\title{
Development of a Virtual Frisch-Grid CZT Detector Based on the Array Structure
}

\author{
Younghak Kim', Wonho Lee ${ }^{2}$ \\ ${ }^{1}$ Department of Bio-convergence Engineering, Korea University, Seoul, Korea; ${ }^{2}$ School of Health and Environmental Science, Korea University, Seoul, Korea
}

\section{Original Research}

Received February 12, 2020

Revision March 10, 2020

Accepted March 10, 2020

Corresponding author: Wonho Lee

School of Health and Environmental Science, Korea University, 145 Anam-ro, Seongbuk-gu, Seoul 02841, Korea

Tel: $+82-2-3290-2754$

Fax: +82-2-3290-5687

E-mail:wonhol@korea.ac.kr

This is an open-access article distributed under the terms of the Creative Commons Attribution License (http://creativecommons.org/licenses/by-nc/4.0), which permits unrestricted use, distribution, and reproduction in any medium, provided the original work is properly cited.

Copyright $\odot$ 2020The Korean Association for Radiation Protection

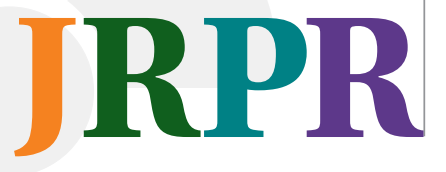

Background: Cadmium zinc telluride (CZT) is a promising material because of a high detection efficiency, good energy resolution, and operability at room temperature. However, the cost of CZT dramatically increases as its size increases. In this study, to achieve a large effective volume with relatively low cost, an array structure comprised of individual virtual Frisch-grid CZT detectors was proposed.

Materials and Methods: The prototype consisted of $2 \times 2 \mathrm{CZTs}$, a holder, anode and cathode printed circuit boards (PCBs), and an application-specific integrated circuit (ASIC). CZTs were used and the non-contacting shielding electrode method was applied for virtual Frisch-grid effect. An ASIC was used, and the holder and the PCBs were fabricated. In the current system, because the CZTs formed a common cathode, a total of 5 channels were assigned for data processing.

Results and Discussion: An experiment using ${ }^{137} \mathrm{Cs}$ at room temperature was conducted for 10 minutes. Energy and timing information was acquired and the depth of interaction was calculated by the timing difference between the signals of both electrodes. Based on obtained three-dimensional position information, the energy correction was carried out, and as a result the energy spectra showed the improvements. In addition, a Compton image was reconstructed using the iterative method.

Conclusion: The virtual Frisch-grid CZT detector based on the array structure was developed and the energy spectra and the Compton image were successfully acquired.

Keywords: Virtual Frisch-Grid CZT, Array Structure, Spectroscopy, Compton Imaging, Radiation Monitoring

\section{Introduction}

Since cadmium zinc telluride (CZT) has several advantages including a high detection efficiency, good energy resolution and operability at room temperature without cooling device, it is a promising material for gamma-ray detection [1]. However, the weighting potential of a CZT detector with planar electrode is linearly distributed with its depth and it means that the amplitude of induced signals depends on the interaction position [2]. The incomplete charge collection due to trapping also occurs, because of low mobility and short lifetime of its hole. Therefore, in general, a detector applying a small pixel structure, coplanar grid and virtual Frisch-grid have been developed instead of the planar electrode [3-5].

Among them, the Polaris-H (H3D Inc., Ann Arbor, MI, USA) and the Rena-Mini long 
(Kromek, Durham, UK) were typical commercialized products. They showed good spectroscopic performances and were able to reconstruct Compton images by using three-dimensional (3D) position and energy information [6]. However, due to the structure based on a large single crystal, there is a limitation that the cost dramatically increased as increase of the crystal size. To overcome this problem, an array type detector which was comprised of individual virtual Frisch-grid CZTs was proposed [7-9]. Those small but high yielding components make the total price lower than that of monolithic detectors. The array structure also has the advantages in the achievement of large effective area by adding the elements and the maintenance of the detector performance by replacing the faulty parts.

In this study, we fabricated a prototype of CZT array detection system which consisted of virtual Frisch-grid CZTs, a detector holder, anode/cathode boards and an application specific integrated circuit (ASIC). Redlen CZT crystals (Redlen Technologies, Saanichton, Canada) and a RenaMini short with embedded RENA-3 ASIC (Kromek) were used $[10,11]$. The detector holder and boards were designed to be compatible with them. The energy spectra were acquired to evaluate the performance. In addition, to verify the feasibility as radiation monitoring device for laboratories, nuclear power plants in decommissioning, etc., the weighted

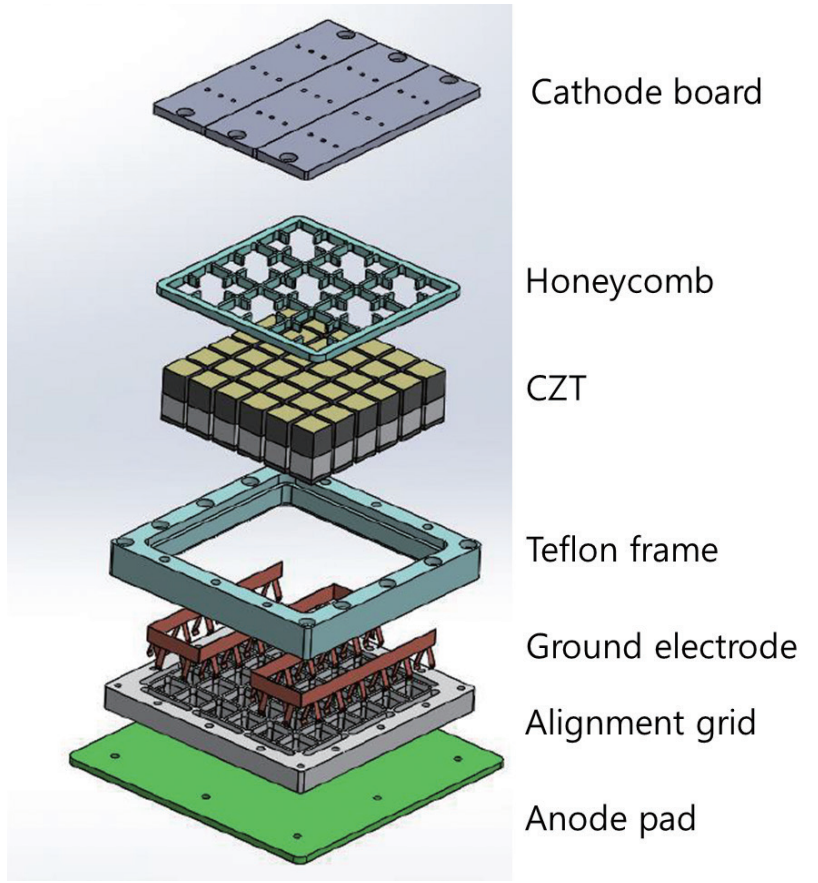

Fig. 1. The schematic of the array module. CZT, cadmium zinc telluride. list-mode maximum likelihood expectation maximization (MLEM) method was applied to the output data and the Compton images were obtained. In case of the imaging, simulations were also carried out to understand the results. The all experiments and simulations were conducted using a ${ }^{137}$ Cs point source.

\section{Materials and Methods}

\section{Array Module}

Fig. 1 shows the schematic of the array module. The prototype was designed based on a $6 \times 6$ array of the $5 \times 5 \times 12 \mathrm{~mm}^{3}$ CZTs and its pixel pitch was determined to be $7.1 \mathrm{~mm}$ considering the CZT size and a fabricable septal thickness of the holder. Fig. 2 shows the manufactured holder and special electrodes. The detector holder which was made of Teflon, an insulating material, consisted of a honeycomb, a frame, an alignment grid for arranging the CZT detectors. The thicknesses of each part were $0.5 \mathrm{~mm}, 7 \mathrm{~mm}, 5 \mathrm{~mm}$, respectively, and because the honeycomb was combined into a groove of the frame, the total thickness was same as that of the CZT. Spring type and ground electrodes were also fabricated by using 0.1-mm-thick beryllium copper ( $\mathrm{CuBe})$. The former were attached to the anode and cathode surfaces of CZT detector by using conductive epoxy for good contacts with the detector boards and sufficient resistance to external shocks. The latter was inserted between the frame and alignment grid to apply the voltage to a shielding electrode of virtual Frisch-grid CZTs.

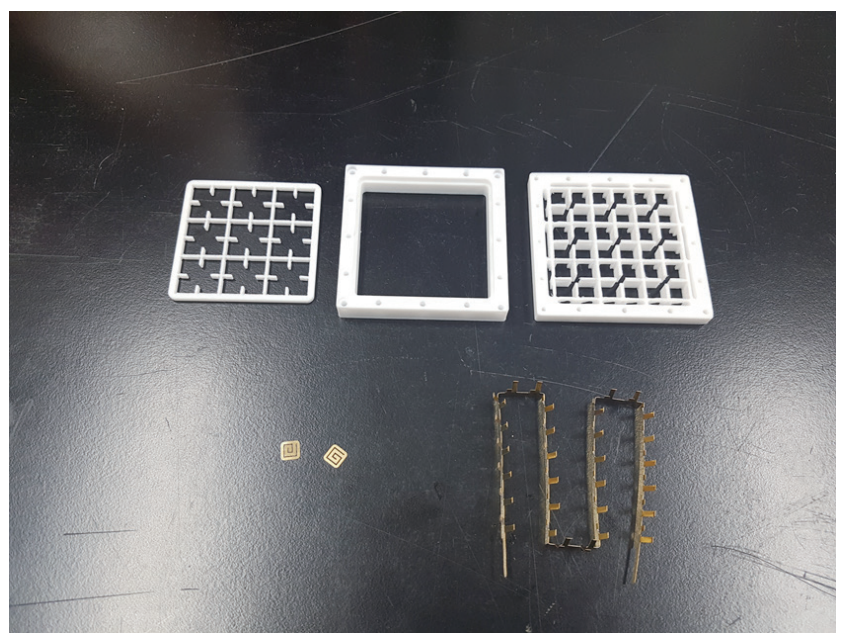

Fig. 2. Photograph of the fabricated honeycomb, frame, alignment grid, spring type, and ground electrodes. 


\section{Data Acquisition System}

Fig. 3 shows the Rena-Mini short with dimensions of $46 \mathrm{~mm} \times 80 \mathrm{~mm} \times 98 \mathrm{~mm}$. The device could be powered by a USB cable and provide the detector boards with high voltage up to 2,000 V. There were two built-in ASIC chips which had 36 independent channels for data processing. Since each channel has two signal paths, one with a fast shaper filter for timing and the other with a slow shaper filter for energy measurement, the both information could be measured, simultaneously. Moreover, the detail options as presented in Table 1 could be easily set via its software according to input signals and a type of detector. The input was digitized by a comparator with 8-bit threshold digital-analog converter and the output was transferred to PC. Power consumption was $6 \mathrm{~mW}$ or less per channel and a tolerance for the direct current (DC) leakage current was up to $5 \mathrm{nA}$.

The detector boards were customized by Nova $R \& D$, a subsidiary of Kromek, taking into account low electric noise, maximum applied voltage and resistance of the CZT (cf. Fig. 4). The electrodes on the anode board consisted of 36 while those on the cathode board formed a common electrode per $2 \times$ 2 CZT array to reduce the necessary number of the channels. Every electrode was completely separated to minimize the crosstalk and the alternating current (AC) coupling was applied for protecting the circuit from the DC current.

\section{Virtual Frisch-Grid CZT}

The non-contacting shielding electrode method was ap-
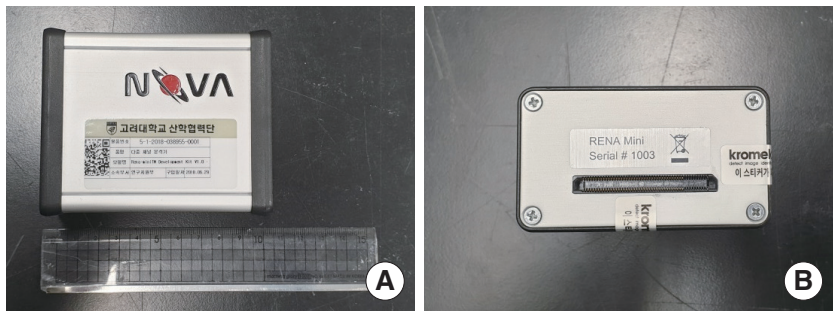

Fig. 3. The front (A) and side surface (B) of Rena-Mini short.

Table 1. Selectable Options of Rena-Mini Short

\begin{tabular}{lc}
\hline Parameter & Value \\
\hline Shaping time $(\mu \mathrm{s})$ & $0.29-38$ \\
Gain & $1.6,1.8,2.3,5$ \\
Feedback resistor $(\mathrm{M} \Omega)$ & $200,1,200$ \\
Feedback capacitor (fF) & 15,60 \\
Polarity & Positive/Negative \\
Threshold & $0-255$ \\
Pole zero cancellation & Enable/Disable \\
\hline
\end{tabular}

plied for the virtual Frisch-grid effect [9] and the specifications of the used Redlen CZT are presented in Table 2. First of all, the side surfaces of each detector were wrapped by an ultra-thin polyethylene terephthalate hot shrink tube which has a dielectric strength over $150 \mathrm{~V} / \mu \mathrm{m}$. Then, $1 / 3$ of them closet to anode were covered with an aluminum tape to form the shielding electrode. Fig. 5 shows CZT detector before and after applying the virtual Frisch-grid method.

In general CZT detectors, the depth of the interaction can be inferred by the electron cloud drift time which indicates the time difference between the signals from the cathode and the anode. Since it was possible to measure the timing information of the both electrodes in current system, the 3D position sensing could be achieved by combining the acquired depth and the position information from the responded pixel. In this study, the spatial resolution determined by the pixel size was $5 \mathrm{~mm}$ in the $\mathrm{x}$, $\mathrm{y}$-direction and that in $\mathrm{z}$-direction was $0.75 \mathrm{~mm}$, because each detector was virtually divided into 20 bins along the depth. The 3D posi-
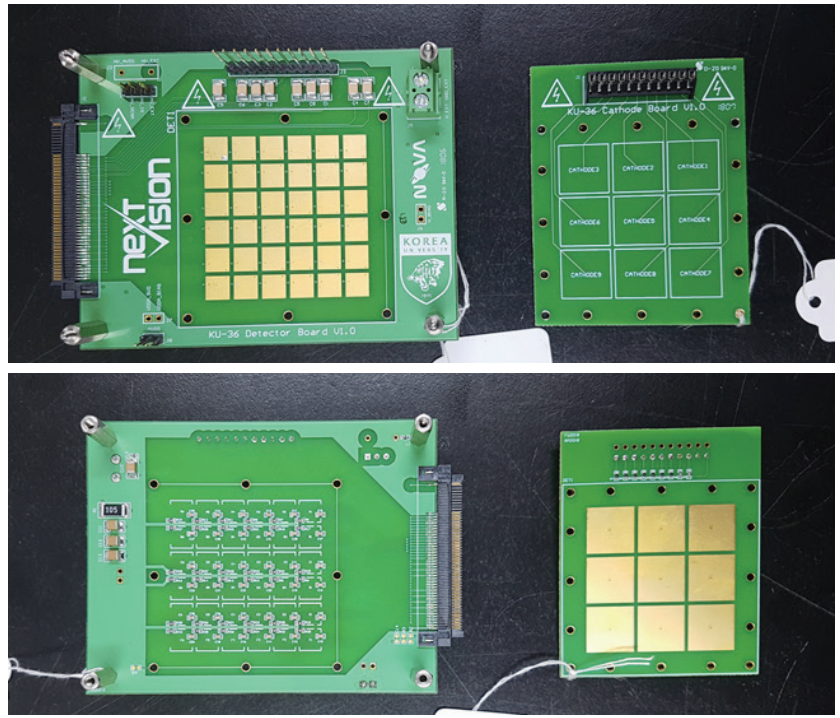

Fig. 4. Photograph of front and rear of the customized anode (left) and cathode (right) boards.

Table 2. Specification of the Redlen CZT

\begin{tabular}{lc}
\hline Parameter & Value \\
\hline Specific inductive capacity & 10.5 \\
Capacitance $(\mathrm{pF})$ & 0.19 \\
Resistivity $(\Omega \cdot \mathrm{cm})$ & $\approx 5 \times 10^{10}$ \\
Dimension $\left(\mathrm{mm}^{3}\right)$ & $5 \times 5 \times 12$ \\
Resistance $(\Omega)$ & $2.4 \times 10^{11}$ \\
Leakage at $1,200 \mathrm{~V}(\mathrm{nA})$ & 5 \\
\hline
\end{tabular}

CZT, cadmium zinc telluride. 


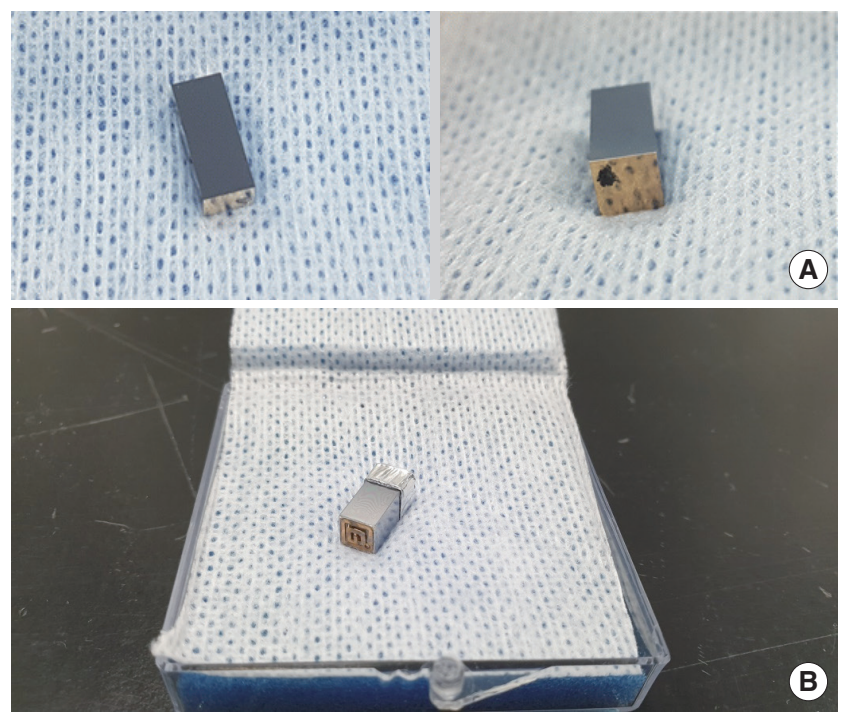

Fig. 5. Photographs of the cadmium zinc telluride (CZT) detector before $(A)$ and after $(B)$ applying the virtual Frisch-grid method.

tion was used to correct the position dependency of the signal amplitude and reconstruct the Compton images.

\section{Image Reconstruction}

When Compton scattering and photoelectric absorption sequentially occur, the incident direction of the gamma-ray can be reconstructed via Compton imaging technique. For Compton imaging, a scattering angle and an axis of each event are required. The scattering angle $\theta$ is calculated by the measured energy as follows:

$$
\cos \theta=1-\frac{m_{e} c^{2} E_{1}}{E_{0}\left(E_{0}-E_{1}\right)}
$$

where $E_{0}$ is the incident energy, $E_{1}$ is the energy deposited in the first interaction, $m_{e}$ is the electron mass, and $c$ is the speed of light. The axis is defined by extrapolating from the second to the first interaction position. Then, a cone based on the scattering angle, the axis, the location of the first interaction can be back-projected on the source plane as shown in Fig. 6. After repeating the back-projections of many events, the overlap of the cones indicates the source position.

To make the source distribution more precise, the weighted list-mode MLEM [12], iterative method based on the statistical model, was applied as described in Equation (2).

$$
\lambda_{j}^{n+1}=\frac{N \lambda_{j}^{n}}{\sum_{i}^{N} Y_{i} \sum_{i}^{N} c_{i j}} \sum_{i}^{N} \frac{Y_{i} c_{i j}}{\sum_{k}^{M} c_{i k} \lambda_{k}{ }^{n}}
$$

where $\lambda_{j}{ }^{n}$ and $\lambda_{j}{ }^{n+1}$ are the current and new estimates, respectively, of the pixel $j$ in the source plane for the $n$-th itera-

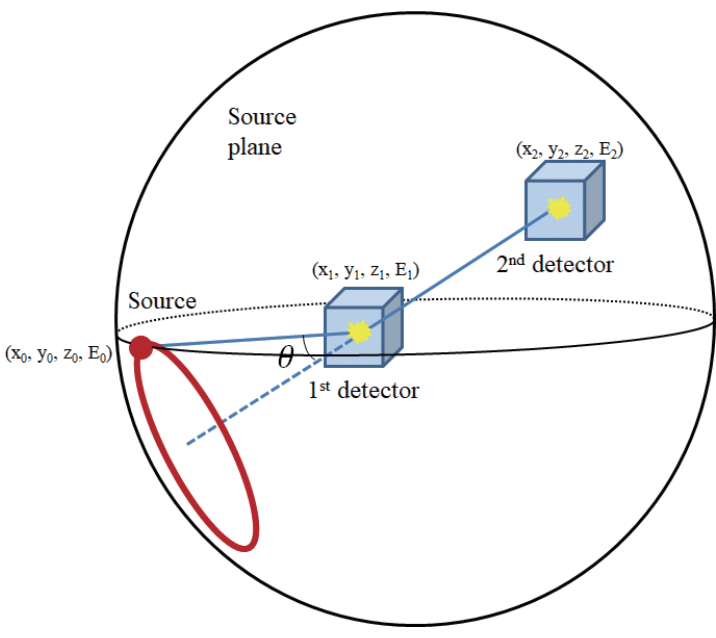

Fig. 6. Schematic of the Compton imaging technique.

tion, $N$ is the number of detected events and $Y_{i}$ is the measured number of counts as event $i$. The event $i$ was defined by information of the scatter detector element, the absorption detector element, and the scattering energy [13]. $c_{i j}$, called the system matrix, is the probability that a photon emitted at pixel $j$ is detected as event $i$. It was calculated considering the probabilistic angular distribution of the Compton scattering, the distances between the interaction positions and the radiation attenuation in the detectors.

In weighted list-mode MLEM, theoretically calculated total angular uncertainty of event $i, \Delta \theta_{i}$ was added to $Y_{i}$ as follows:

$$
Y_{i}=\frac{1}{\Delta \theta_{i}}=\frac{1}{\sqrt{\Delta \theta_{\text {energy }}^{2}+\Delta \theta_{\text {geometry }}^{2}+\Delta \theta_{\text {Doppler }}^{2}}}
$$

where $\Delta \theta_{\text {energy }}^{2}, \Delta \theta_{\text {geometry, }}^{2}$ and $\Delta \theta_{\text {Doppler }}^{2}$ were the angular uncertainties caused by energy uncertainty, voxel size, and Doppler effects, respectively. As shown Equations (2) and (3), the contribution of each event is inversely proportional to its total angular uncertainty. Since this uncertainty could be increased by increasing the number of scatterings, only twointeraction events were used for the image reconstruction.

\section{Experiment Setup}

The array module was assembled with four CZT detectors as shown in Fig. 7 and connected to the ASIC in an aluminum faraday box to block external electromagnetic fields. Fig. 8 shows the photographs and cross-section of the experiment setup. The $650 \mathrm{kBq}{ }^{137} \mathrm{Cs}$ source was positioned $5 \mathrm{~cm}$ away from the center of the array and was measured at room temperature for 10 minutes. The anode and the shielding 

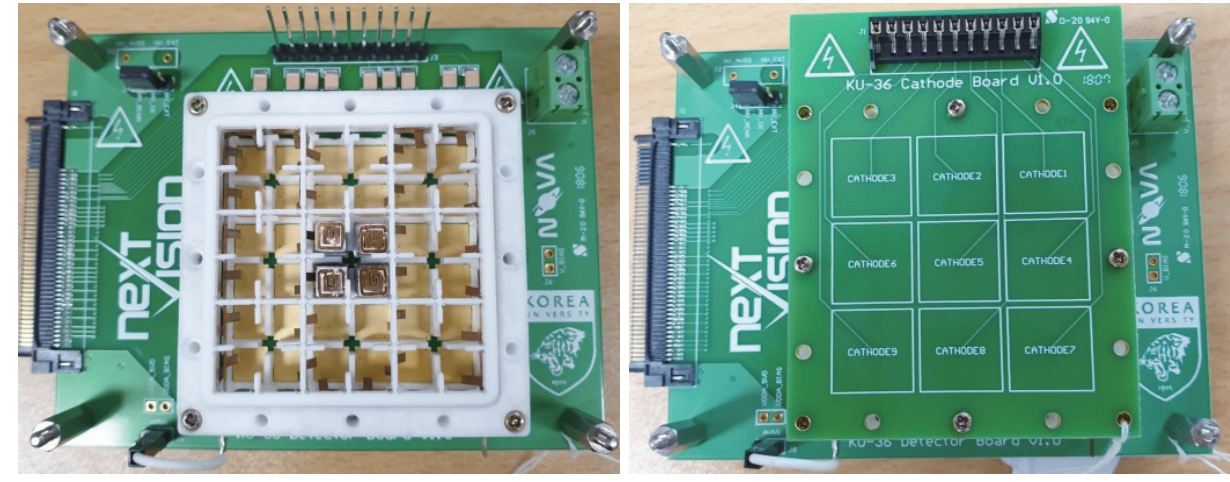

Fig. 7. The photographs of the assembled array module.
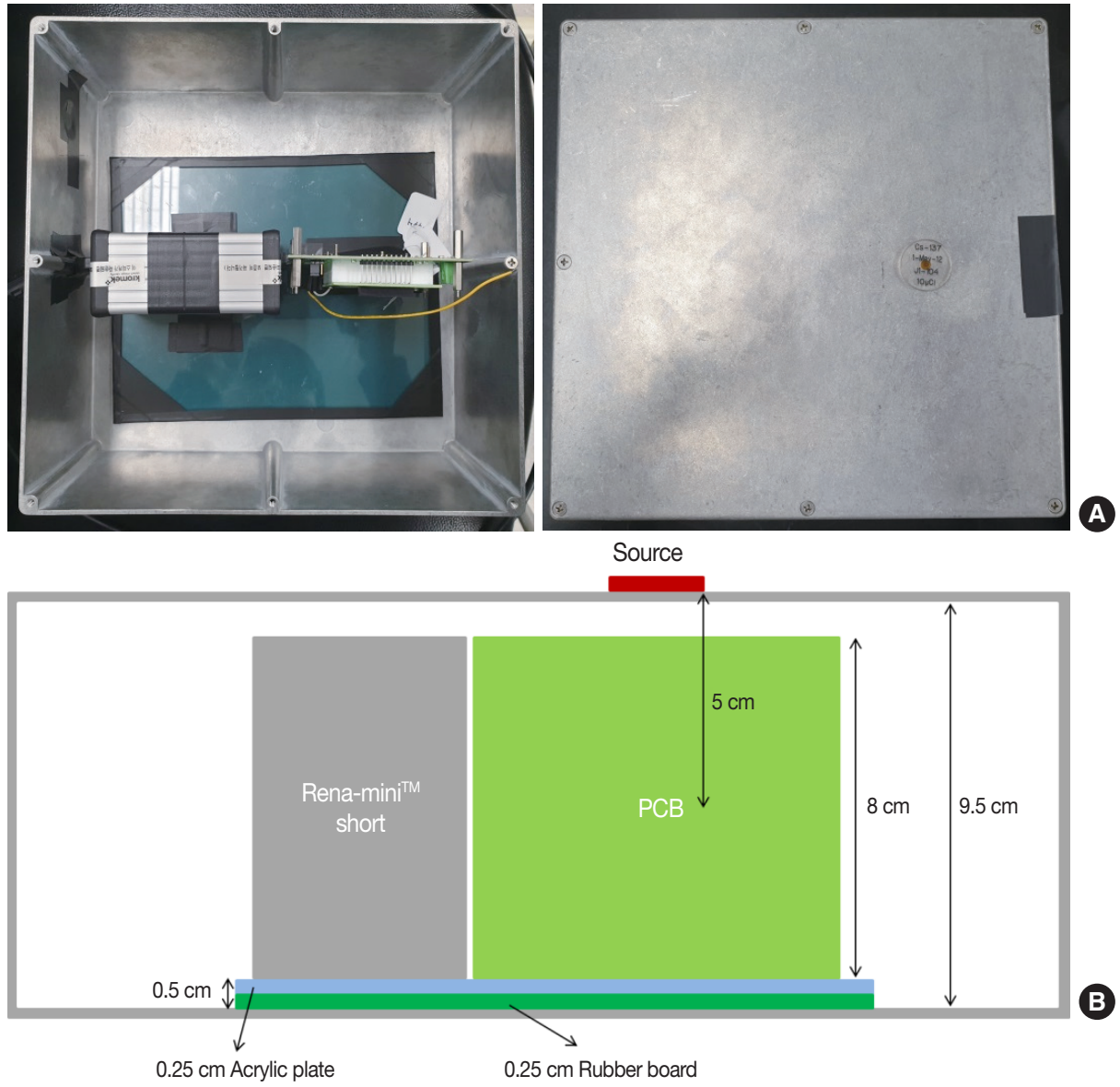

Fig. 8. The photographs (A) and cross-section (B) of the experimental setup.

electrode were grounded, and the negative high voltage was applied to the cathode. Since a $100 \mathrm{~V} / \mathrm{mm}$ or less bias was usually applied to prevent the electronic noise, the cathode's bias was set at $-1,200 \mathrm{~V}$. The threshold and gain were chosen appropriately and the shaping time of the anode and the cathode were set to $1.1 \mu$ s and $2.3 \mu$ s, respectively.

\section{Results and Discussion}

\section{Spectroscopy}

Fig. 9 represents the pulse height spectra of the triggered events on anode. Each CZT detector showed different performances but represented a general shape of uncorrected spectrum. The energy resolutions before correction were 

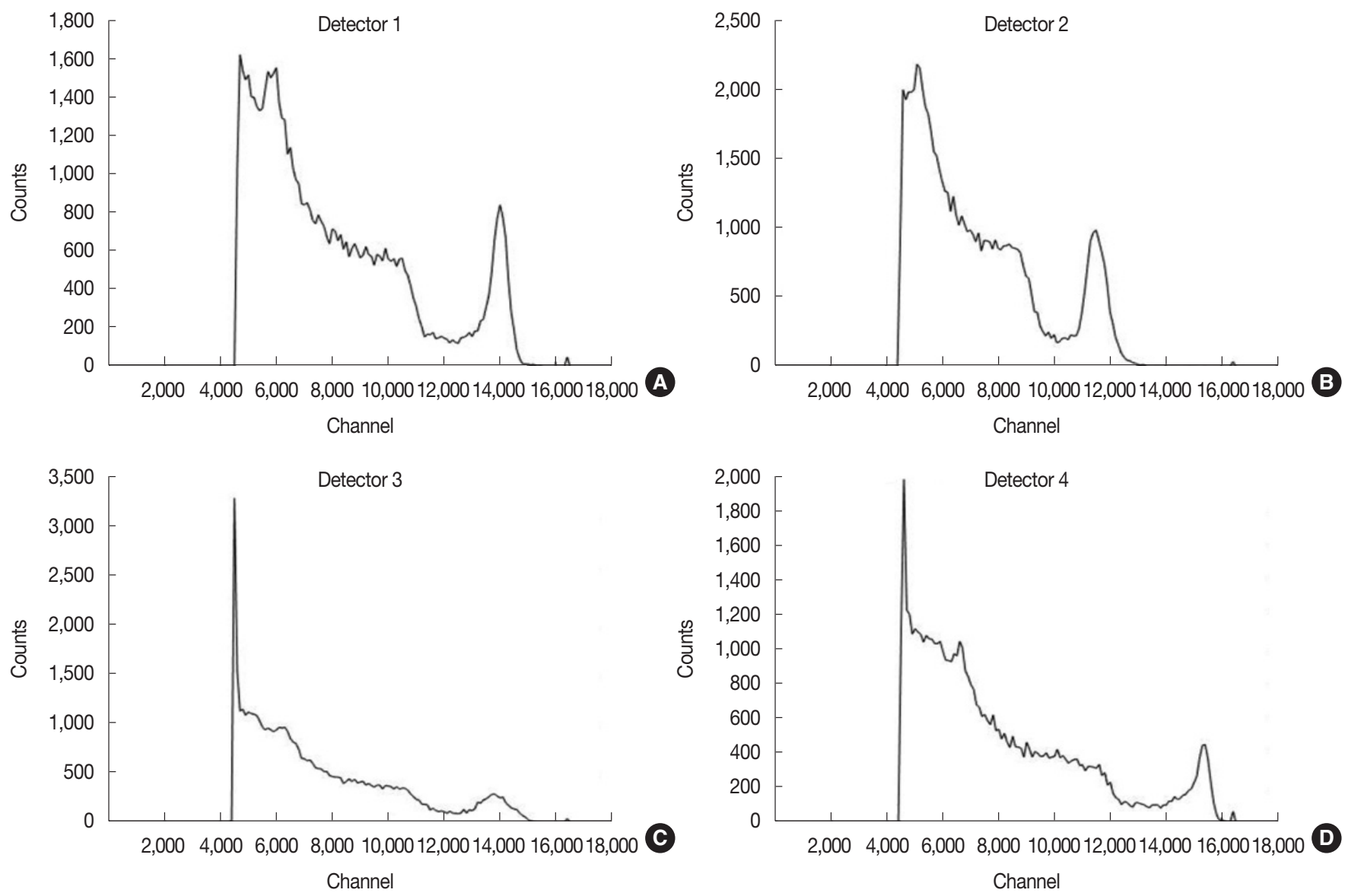

Fig. 9. The pulse height spectra of all triggered events on anode: (A) detector 1, (B) detector 2, (C) detector 3, and (D) detector 4.

$5.8 \%, 8.6 \%, 12.8 \%$, and $6.5 \%$ at $662 \mathrm{keV}$, respectively. To applying correction method, we extracted the events that both electrodes were triggered at the same time and acquired the 3D position information. Then, the correction was applied by multiplying the signal amplitude with a correction factor corresponding to the position where the signal occurred. The factor was a ratio of the peak channel of the total spectrum to that of each voxel. After the correction, the overall results showed the improvements as shown in Fig. 10. The photopeaks became sharper and the peak-to-Compton ratio which was the height of the photopeak divided by the height of the Compton continuum in spectrum increased. The energy resolutions were $4.6 \%, 9.1 \%, 9.0 \%$, and $4.5 \%$ which were improved by $20 \%$ on average. However, owing to the intrinsic quality difference of the CZT crystal and the insufficient correction attributed to short measurement time, there were still the differences between the detectors. In addition, the low energy region of each spectrum was suppressed. This is because that there was a $15 \mathrm{kHz}$ ripple noise in the cathode output and therefore its threshold was set high to remove it.
We guessed that this noise was due to an incomplete rectification and a stable external DC high voltage supplier became a solution.

\section{Compton Imaging}

To determine the sequence order of Compton events, the simple comparison method based on the deposit energies of each interaction was used. Since the probability that a photon deposits more energy in the Compton scattering is affected by energy and an incident direction of the gammaray, a detector material and geometry, it depends on a detection system and situations. If the sequence orders of Compton events were wrong, they caused some artifacts on the opposite of source position or other sides on source plane. To determine the sequence order correctly, the simulations were preceded. The simulation tool was Geant4 Application for Tomographic Emission (GATE) v7.0. The detector structure was set up identically to the experiment and sources with $0^{\circ}-90^{\circ}$ offset were simulated. As a result, the ratio of deposit more energy in the Compton scattering was about $76 \%$ 

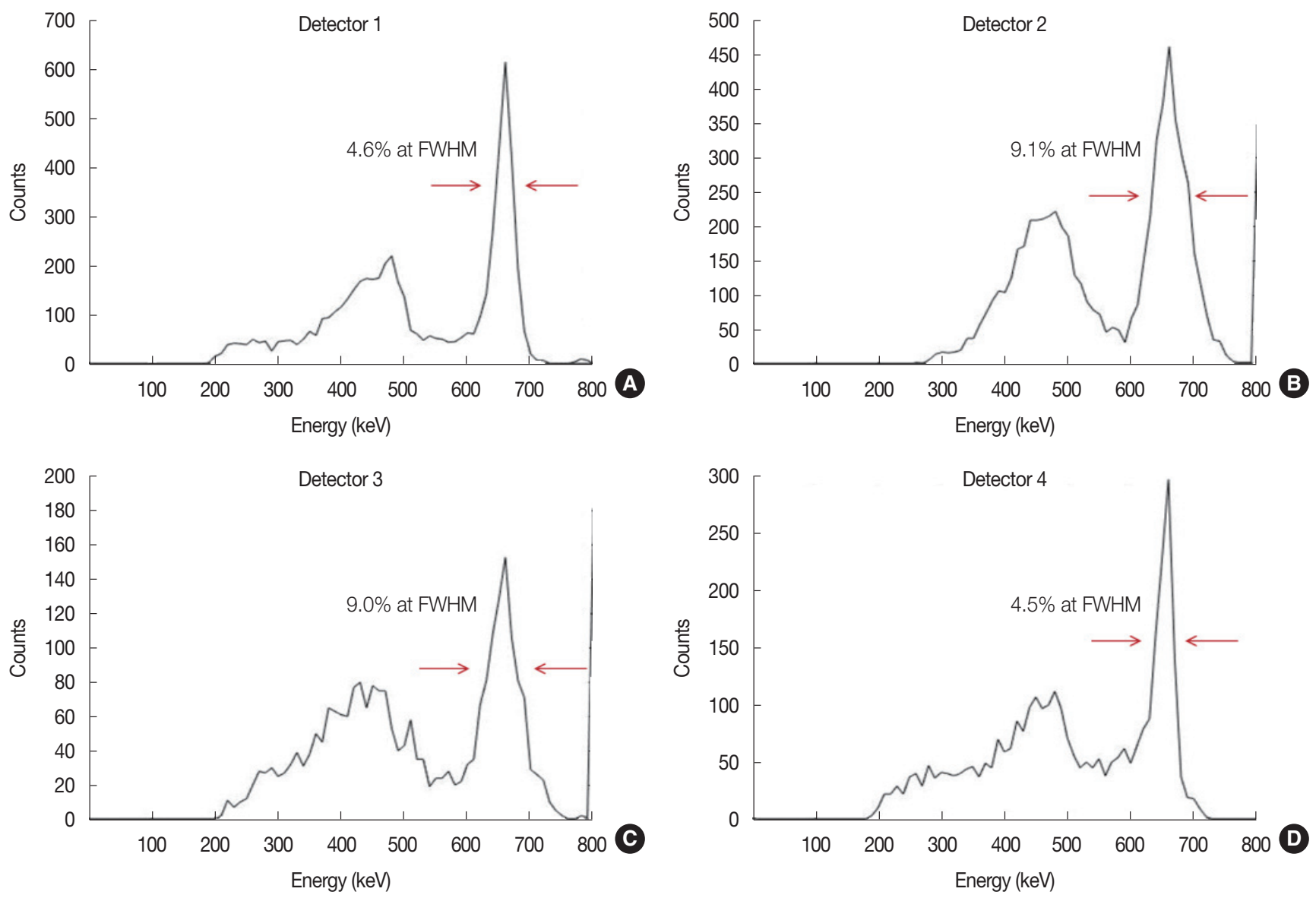

Fig. 10. The corrected energy spectra of events that both anode and cathode were triggered: (A) detector 1, (B) detector 2, (C) detector 3, and (D) detector 4. FWHM, full width at half maximum.

for all positions of sources, so we regarded the interaction delivering more energy as the first interaction [14].

Fig. 11 represents the planar Compton images of simulations based on the correct and opposite sequence orders and the number of iteration was 5 . In case of the correct sequence order, each source was well located at its own position. However, because the measurable detection positions were very limited in the $\mathrm{x}$ and $\mathrm{y}$ directions, and the spatial resolutions of them were different from depth, the distributions showed the distortions according to increase offset angle. This angular dependency could be mitigated by adding the CZT components because the detectable position in $\mathrm{x}$ and y directions became various. In result of the wrong sequence order, the large artifacts appeared near the source and it makes the source difficult to distinguish. Fig. 12 shows the reconstructed $4 \pi$ and planar images in the experiment. The configuration of detection system and source was same as that of the $90^{\circ}$ offset source simulation. Compared the simulations, the result was similar but the artifact was domi- nant due to lack of the effective events. Table 3 shows a quantitative evaluation of the current system and a comparison of the Polaris-H, the Rena-Mini long, and a virtual Frisch-grid detector array from Brookhaven National Laboratory. This result represented that the current system needed to be developed, and if the entire system is completed, it is expected to be competitive with other systems.

\section{Conclusion}

In this study, the virtual Frisch-grid CZT detector based on $2 \times 2$ array structure was developed and the experiment of ${ }^{137} \mathrm{Cs}$ was performed. The energy and timing information of both the anode and cathode was acquired at the same time. Since it is possible to detect 3D position by calculating the drift time, the variations of signal amplitude were corrected in accordance with the interaction position. As a result, the energy spectra were improved but the low energy regions were not formed properly. This is because that the threshold 

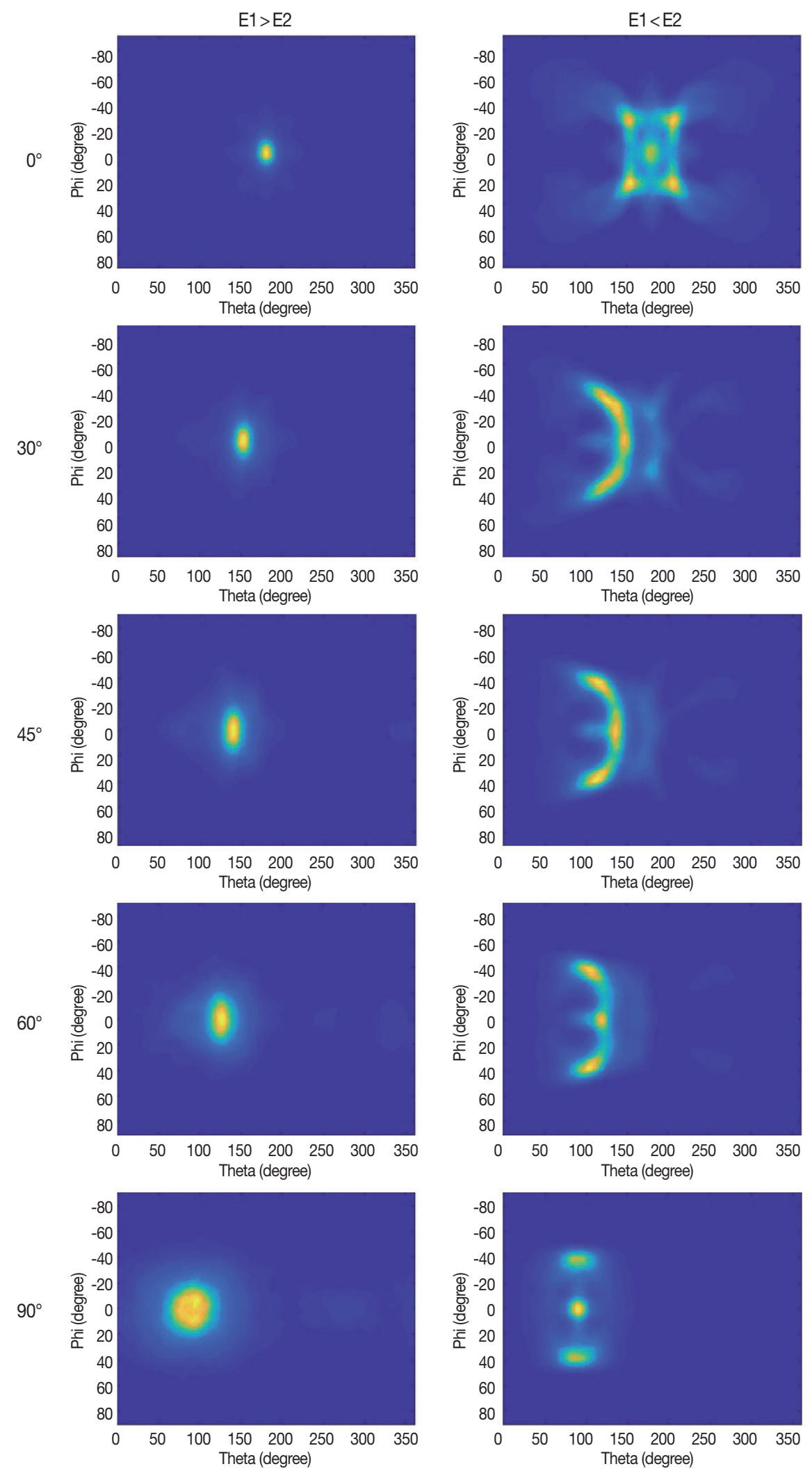

Fig. 11. The planar Compton images of ${ }^{137} \mathrm{Cs}$ with offset angles in simulation ( $5^{\text {th }}$ iteration) based on the correct (left)/reverse (right) sequence order. 

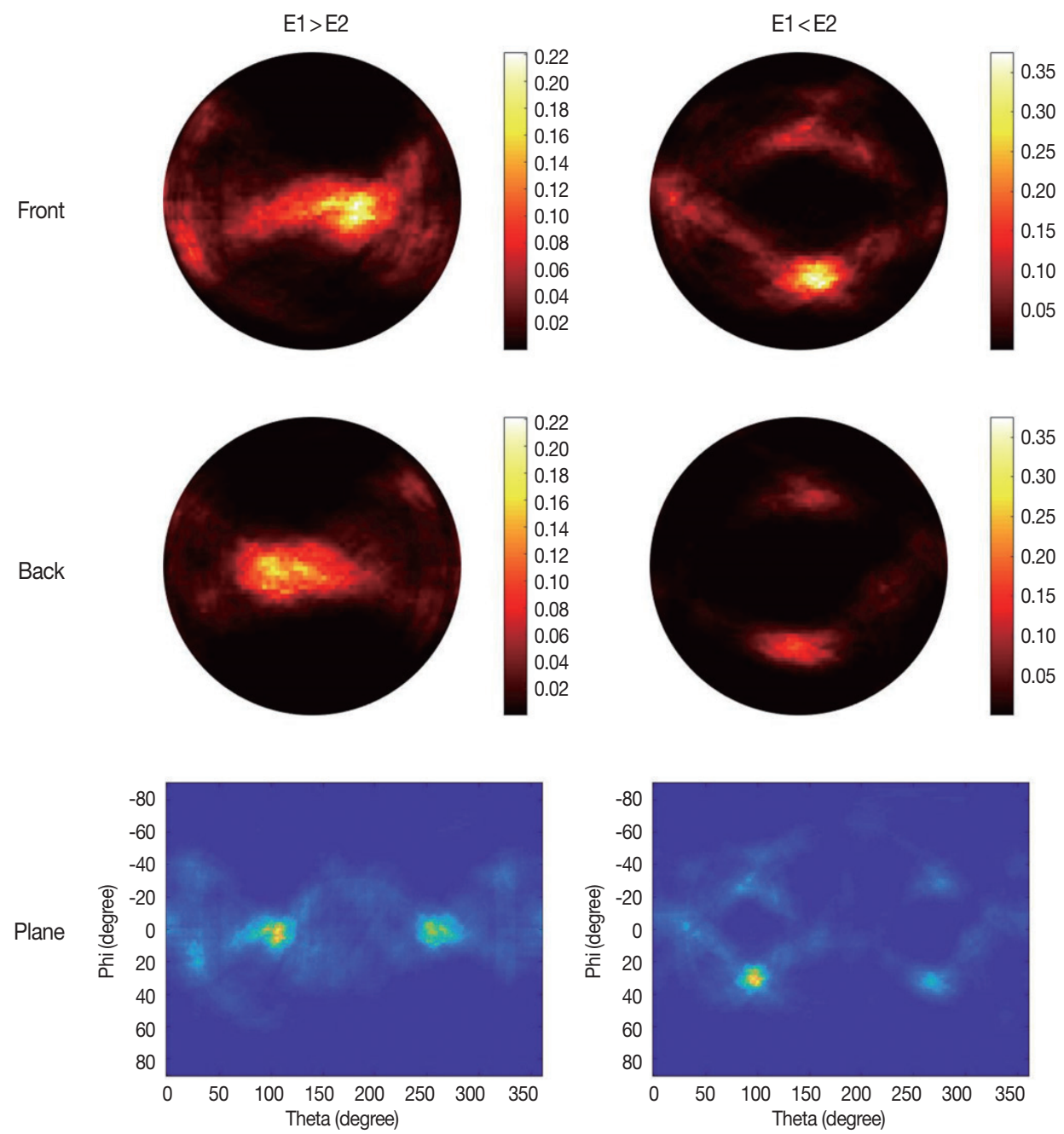

Fig. 12. The $4 \pi$ and planar Compton images of ${ }^{137} \mathrm{Cs}$ with $90^{\circ}$ offset angle in experiment ( $5^{\text {th }}$ iteration) based on the correct (left)/reverse (right) sequence order.

Table 3. Comparison of Performances for a 662 keV Radiation [6]

\begin{tabular}{|c|c|c|c|c|c|}
\hline \multirow{2}{*}{ System } & \multirow{2}{*}{ Electrode } & \multicolumn{2}{|c|}{ Size $\left(\mathrm{mm}^{3}\right)$} & \multirow{2}{*}{$\begin{array}{l}\text { Intrinsic efficiency } \\
\text { for imaging }\end{array}$} & \multirow{2}{*}{$\begin{array}{l}\text { Angular resolutior } \\
\text { (iteration) }\end{array}$} \\
\hline & & CZT & Pixel & & \\
\hline Polaris-H100 (H3D Inc.) & Pixelated anode & $20 \times 20 \times 15$ & $1.22 \times 1.22 \times 15$ & $\sim 2.00 \times 10^{-2}$ & $\sim 20^{\circ}(-)$ \\
\hline Rena-Mini long (Kromek) & Pixelated anode & $20 \times 20 \times 5$ & $2.5 \times 2.5 \times 5$ & $6.70 \times 10^{-4}$ & $10.18^{\circ}(10)$ \\
\hline Vritaul Frisch-grid detector array (BNL) & Virtual Frisch-grid & $36 \times 36 \times 15$ & $6 \times 6 \times 15$ & $9.40 \times 10^{-3}$ & $26 \pm 1.30^{\circ}(10)$ \\
\hline Virtual Frisch-grid detector array (current system) & Virtual Frisch-grid & $10 \times 10 \times 12$ & $5 \times 5 \times 12$ & $2.00 \times 10^{-4}$ & $18.85^{\circ}(5)$ \\
\hline
\end{tabular}

CZT, cadmium zinc telluride; BNL, Brookhaven National Laboratory.

of the cathode to eliminate the rippling noise was too high. To resolve this problem, the stable external DC high voltage supplied will be used in the future.

To identify the viability as a Compton imager, the weighted list-mode MLEM was applied to the output data. The simulations with various offset source were also performed and the sequence orders of the effective Compton events were determined by the deposit energies. In simulation results, the sources were well located at each offset angle but the performance was degraded with increasing of the offset angle due to the different spatial resolution $\mathrm{x}, \mathrm{y}$ direction and depth. The Compton image of the experiment was successfully acquired and similar to that of the simulation. 


\section{Conflict of Interest}

No potential conflict of interest relevant to this article was reported.

\section{Acknowledgements}

This work was supported by the Nuclear Safety Research Program through the Korea Foundation of Nuclear Safety (KoFONS) using the financial resource granted by the Nuclear Safety and Security Commission (NSSC) of the Republic of Korea (No. 1903006).

\section{References}

1. Sordo SD, Abbene L, Caroli E, Mancini AM, Zappettini A, Ubertini P. Progress in the development of CdTe and CdZnTe semiconductor radiation detectors for astrophysical and medical applications. Sensors (Basel). 2009;9:3491-3526.

2. He Z. Review of the Shockley-Ramo theorem and its application in semiconductor gamma-ray detectors. Nucl Instrum Methods Phys Res A. 2001;463:250-267.

3. He Z, Li W, Knoll GF, Wehe DK, Berry J, Stahle CM. 3-D position sensitive CdZnTe gamma-ray spectrometers. Nucl Instrum Methods Phys Res A. 1999;422:173-178.

4. Luke PN. Unipolar charge sensing with coplanar electrodes-application to semiconductor detectors. IEEE Trans Nucl Sci. 1995; 42:207-213.

5. McGregor DS, He Z, Seifert HA, Wehe DK, Rojeski RA. Single charge carrier type sensing with a parallel strip pseudo-Frischgrid CdZnTe semiconductor radiation detector. Appl Phys Lett. 1998;72:792-794.

6. Kim Y, Lee T, Lee W. Radiation measurement and imaging using 3D position sensitive pixelated CZT detector. Nucl Eng Technol.
2019;51:1417-1427.

7. Bolotnikov AE, Camarda GS, Cui Y, Egarievwe SU, Fochuk PM, Fuerstnau M, et al. Array of virtual Frisch-grid CZT detectors with common cathode readout and pulse-height correction. In: Proceedings of SPIE 7805: Hard X-ray, gamma-ray, and neutron detector physics XII. Bellingham, WA: International Society for Optics and Photonics; 2010.

8. Bolotnikov AE, Butcher J, Camarda GS, Cui Y, De Geronimo G, Fried J, et al. Array of virtual Frisch-grid CZT detectors with common cathode readout for correcting charge signals and rejection of incomplete charge-collection events. IEEE Trans Nucl Sci. 2012;59:1544-1551.

9. Bolotnikov AE, Ackley K, Camarda GS, Cherches C, Cui Y, De Geronimo G, et al. An array of virtual Frisch-grid CdZnTe detectors and a front-end application-specific integrated circuit for large-area position-sensitive gamma-ray cameras. Rev Sci Instrum. 2015;86:073114

10. Pike SN, Harrison FA, Burnham JA, Cook WW, Grefenstette BW, Madsen KK, et al. Characterization of Redlen CZT detectors for x-ray astronomy. In: Proceedings of SPIE 10709: High energy, optical, and infrared detectors for astronomy VIII. Bellingham, WA: International Society for Optics and Photonics; 2018.

11. Volkovskii A, Clajus M, Gottesman SR, Malik H, Schwartz K, Tumer E, et al. Design of a coded aperture Compton telescope imaging system (CACTIS). In: Proceedings of SPIE 7805: Hard XRay, Gamma-Ray, and Neutron Detector Physics XII. Bellingham, WA: International Society for Optics and Photonics; 2010.

12. Lehner CE, He Z, Zhang F. $4 \pi$ Compton imaging using a 3-D position-sensitive CdZnTe detector via weighted list-mode maximum likelihood. IEEE Trans Nucl Sci. 2004;51:1618-1624.

13. Xu D. Gamma-ray imaging and polarization measurement using 3-D positionsensitive CdZnTe detectors [dissertation]. Ann Arbor, MI: University of Michigan; 2006.

14. Kim YH, Lee T, Lee W. Double-layered CZT Compton imager. IEEE Trans Nucl Sci. 2017;64:1769-1773. 